\title{
BMJ Open Effects of adenotonsillectomy on the growth of children with obstructive sleep apnoea-hypopnea syndrome (OSAHS): protocol for a systematic review
}

\author{
Fang Hua, ${ }^{\oplus, 2,3}$ Tingting Zhao, ${ }^{1}$ Tanya Walsh, ${ }^{3}$ Qiao Sun, ${ }^{1}$ Xiong Chen, ${ }^{4}$ \\ Helen Worthington, ${ }^{3}$ Fan Jiang, ${ }^{5}$ Hong $\mathrm{He}^{1}$
}

To cite: Hua F, Zhao T, Walsh T, et al. Effects of adenotonsillectomy on the growth of children with obstructive sleep apnoeahypopnea syndrome (OSAHS): protocol for a systematic review. BMJ Open 2019;9:e030866. doi:10.1136/ bmjopen-2019-030866

- Prepublication history for this paper is available online. To view these files, please visit the journal online (http://dx.doi. org/10.1136/bmjopen-2019030866).

FH and TZ contributed equally.

Received 04 April 2019

Revised 16 July 2019

Accepted 19 July 2019

D) Check for updates

(C) Author(s) (or their employer(s)) 2019. Re-use permitted under CC BY-NC. No commercial re-use. See rights and permissions. Published by BMJ.

For numbered affiliations see end of article.

Correspondence to

Hong He;

drhehong@whu.edu.cn

\section{ABSTRACT}

Introduction Obstructive sleep apnoea-hypopnea syndrome (OSAHS) is characterised by recurring episodes of complete or partial upper airway collapse during sleep. Persistent OSAHS is associated with long-term consequences, such as growth failure, cardiovascular and neurocognitive problems in children. Different from the aetiology of OSAHS in adults, the most common cause of paediatric OSAHS is adenotonsillar hypertrophy. Adenotonsillectomy (AT) has been recommended as the first-line treatment of paediatric OSAHS. Several studies have suggested that retarded growth caused by OSAHS can improve after AT during the prepubertal period. This review will systematically search and summarise the available evidence on the effects of AT on children's growth.

Methods and analysis We will conduct electronic searches in MEDLINE (via PubMed), Embase, Google Scholar and the Cochrane Central Register of Controlled Trials for randomised controlled trials (RCTs) or cohort studies that included a control group. Additional records will be searched by checking the references included in the selected studies and relevant reviews. At least two authors will undertake selection of studies and data extraction independently and in duplicate. The Cochrane Risk of Bias tool and Risk Of Bias In Non-randomised Studies - of Interventions will be used to assess the risk of bias of RCT and cohort studies, respectively. A randomeffects model will be used for meta-analyses. Data synthesis and other analyses will be carried out using the RevMan V.5.3 software. The Grades of Recommendation, Assessment, Development and Evaluation will be used to assess the quality of the supporting evidence behind each main comparison.

Ethics and dissemination There is no ethical issue in this systematic review given that we will only include published studies. The results will be disseminated via peer-reviewed publications and social networks. PROSPERO registration number CRD42019125882

\section{INTRODUCTION}

\section{Background}

Obstructive sleep apnoea-hypopnea syndrome (OSAHS) has been defined as 'a
Strengths and limitations of this study

- To our knowledge, this will be the first systematic review regarding the effects of adenotonsillectomy on children's growth since 2009 .

- Both randomised controlled trials and prospective cohort studies will be searched and included (if eligible), which could result in a more comprehensive summary of the available evidence.

- Subgroup and sensitivity analyses will be performed to explore heterogeneity and the robustness of our findings.

- The main limitation might result from a limited number of primary studies that are available, and a potentially low certainty of evidence from observational studies.

- There is a lack of commonly accepted minimal clinically important difference for the outcome measures of interest and this research question, making it potentially difficult to interpret the clinical significance of synthesised results.

disorder of breathing during sleep characterized by prolonged partial upper airway obstruction and/or intermittent complete obstruction that disrupts normal ventilation during sleep and normal sleep patterns'. ${ }^{1}$ The reported prevalence of paediatric OSAHS varies from $0.6 \%$ to $44.6 \%$ depending on the population studied and the definitions/standards used for diagnosis. ${ }^{2-4}$ The commonly considered gold standard for diagnosing paediatric OSAHS is overnight polysomnography (PSG). ${ }^{1}$ Children with OSAHS may show symptoms such as snoring, mouth breathing, observed episodes of apnoea, as well as attention-deficit or hyperactivity. Also, growth retardation, ${ }^{5} 6$ neuropsychological and cognitive deficits, ${ }^{7}$ impaired cardiovascular function ${ }^{89}$ as well as craniofacial development disorders have been reported to be 
associated with OSAHS. ${ }^{10} 11$ However, the pathophysiological mechanism behind poor growth in children with OSAHS is unclear. Possible reasons may include the increased energy expenditure during sleep, abnormal nocturnal growth hormone secretion, nocturnal hypoxemia, nocturnal respiratory acidosis, as well as lower total caloric intake resulting from poor appetite and difficulties in swallowing. ${ }^{12}$

\section{Description of the intervention}

There are a variety of therapies available for the treatment of paediatric OSAHS, including surgical and non-surgical treatments. ${ }^{14-16}$ Adenotonsillar hypertrophy is the most important anatomical factor that has been associated with OSAHS, ${ }^{17}$ therefore adenotonsillectomy (AT) has been widely recommended as the first-line treatment. ${ }^{18}$ For children between 3 and 17 years who are comorbidity free, AT appears to be a safe option with a growing body of evidence. ${ }^{19}$ Nowadays, many paediatric Ears, nose, and throat (ENT) surgeons are using the Coblation technique (cold radiofrequency ablation) and a lower complication rate has been reported. ${ }^{20}$ For clinicians faced with the decision of whether to perform AT, the severity of obstructive sleep apnoea is an important factor. Removal of the enlarged adenoid and tonsil eliminates the upper airway obstruction, improves the breathing pattern and improves the respiratory parameters as measured by PSG in the majority of healthy children. ${ }^{21}$

\section{Why it is important to conduct this review}

Recent studies have suggested that retarded growth caused by OSAHS can improve after AT. ${ }^{12}{ }^{13}{ }^{18} 22-24$ However, there have been few systematic reviews analysing the effects of AT on the growth of children with OSAHS. In 2009, Bonuck et $a l^{25}$ carried out a systematic review and meta-analysis regarding the growth and growth biomarker changes after AT, but did not make a comparison between children who received AT and an appropriate control group. To our knowledge, at present there exists no other systematic reviews regarding this question. We therefore have a unique opportunity to systematically review the relevant literature and determine the effects of AT on children's growth.

\section{Objectives}

The main objective of this review is to assess the effects of AT on the growth of children with OSAHS.

\section{Review question}

Does AT have any effects on the growth of children with OSAHS, as compared with concurrent controls (either children with OSAHS who did not receive AT or healthy controls)?

\section{METHODS}

This protocol was written in accordance with the Preferred Reporting Items for Systematic Review and Meta-Analysis Protocols reporting guidelines. ${ }^{26}$
Criteria for considering studies for this review

Types of studies

We will include randomised controlled trials (RCTs) and prospective cohort studies that:

1. Reported on the growth changes of children before and after AT.

2. Included a concurrent control group.

3. Had a follow-up length of no $<6$ months.

For a prospective cohort study to be eligible in the review it should meet the following criteria: (1) it should include two or more groups of participants; (2) the identification of participants, the assessment of baseline, the allocation to intervention and the assessment of outcomes should be done prospectively. We will not require that the study hypothesis was generated prospectively as this aspect is generally poorly reported. ${ }^{27}$

\section{Types of participants}

We will include studies in which the majority of participants were under 18 years of age at the time of recruitment with a diagnosis of OSAHS.

\section{Types of interventions}

Treatment group

AT (tonsillectomy and/or adenoidectomy)

Control group:

1. No intervention or watchful waiting only

2. Other pharmacological or surgical interventions.

Types of outcome measures

Primary outcome:

1. Height $\mathrm{Z}$ score (continuous outcome, measured at least 6 months after AT with stadiometer).

Secondary outcomes:

1. Raw value of height in cm (continuous outcome, measured at least 6 months after AT with stadiometer).

2. Weight $\mathrm{Z}$ score (continuous outcome, measured at least 6 months after AT with electronic scale).

3. Raw value of weight in $\mathrm{kg}$ (continuous outcome, measured at least 6 months after AT with electronic scale).

4. Body Mass Index (BMI) Z score (continuous outcome, calculated based on height and weight data meeting the above-mentioned criteria).

5. BMI (continuous outcome, calculated based on height and weight data meeting the above-mentioned criteria).

6. Insulin-like growth factor-1 (IGF-1), Insulin-like growth factor binding protein-3 (IGFBP-3), growth hormone, concentration $\mathrm{Z}$ score (continuous outcome, measured at least 6 months after AT using blood samples and chemiluminescence ELISA).

7. IGF-1, IGFBP-3, growth hormone, concentration in $\mathrm{ng} / \mathrm{mL}$ (continuous outcome, measured at least 6 months after AT using blood samples and chemiluminescence ELISA). 


\begin{tabular}{|c|c|}
\hline No & Search terms \\
\hline \#1 & "Tonsillectomy"(Mesh) \\
\hline \#2 & "Palatine Tonsil/surgery"(Mesh) \\
\hline \#3 & $\begin{array}{l}\text { adenotonsillecto* OR adenotonsilectom* }{ }^{*} \text { OR } \\
\text { tonsillecto*OR tonsillotom }{ }^{\star} \text { OR adenoidectom* } \\
\text { OR tonsilotom* }\end{array}$ \\
\hline \#4 & 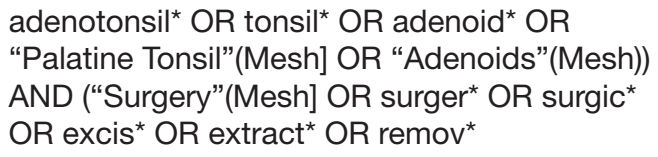 \\
\hline \#5 & \#1 OR \#2 OR \#3 OR \#4 \\
\hline \#6 & “Sleep Apnoea Syndromes”(Mesh) \\
\hline$\# 7$ & sleep* AND (apnea* OR apnoea*) \\
\hline \#8 & hypopnea* OR hypopnoea* \\
\hline \#9 & OSA OR OSAHS \\
\hline$\# 10$ & \#6 OR \#7 OR \#8 OR \#9 \\
\hline \#11 & child(Mesh] OR adolescent(Mesh) \\
\hline \#12 & $\begin{array}{l}\text { child }^{\star} \text { OR adolescent* OR pediatric* OR } \\
\text { paediatric* }\end{array}$ \\
\hline \#13 & $\begin{array}{l}\text { child }^{\star}\left(\text { Title/Abstract)OR adolescent }{ }^{\star}(\text { Title/ }\right. \\
\text { Abstract)OR pediatric*(Title/Abstract)OR } \\
\text { paediatric*(Title/Abstract) }\end{array}$ \\
\hline \#14 & \#5 AND \#10 AND (\#11 OR \#12 OR \#13) \\
\hline \#15 & $\begin{array}{l}\text { review(publication type] OR "systematic } \\
\text { review"(publication type] OR "meta } \\
\text { analysis"(publication type] OR "case } \\
\text { reports"(publication type] OR letter(publication } \\
\text { type] OR editorial(publication type] OR } \\
\text { comment(publication type) }\end{array}$ \\
\hline \#16 & \#14 NOT \#15 \\
\hline
\end{tabular}

\section{Search strategy}

\section{Electronic searches}

We will search MEDLINE (via PubMed), Embase, Google Scholar and the Cochrane Central Register of Controlled Trials with no restrictions on language or publication date. The detailed search strategy for MEDLINE is listed in table 1 , which will be adapted to the syntax and subject headings of the other databases.

\section{Searching other resources}

Additional studies will be sought by manually checking the references of included studies and relevant review articles.

\section{Data collection and analysis}

We will follow the relevant guidance provided in the Cochrane Handbook for Systematic Reviews. ${ }^{28}$

\section{Study selection}

At least two authors will screen the titles and abstracts yielded from the literature searches, independently and in duplicate. Articles, which appear to meet our inclusion criteria, will be downloaded in full. The same group of authors will then examine these full-texts independently for eligibility. Any disagreement will be resolved by discussion and consultation with other authors until consensus is reached. The corresponding author will be contacted if a study has insufficient information. For each excluded study, we will document the reason for exclusion.

\section{Data extraction and management}

The bibliographic information and abstracts of all identified items will be imported into the EndNote V.X8 Software. At least two review authors will independently extract the following data from the included studies:

1. General information: title, journal, year, publication status.

2. Study characteristics: sample size, methods used for randomisation and allocation concealment, blinding.

3. Participants: age, sex, race, height, weight, BMI, IGF-1, IGFBP-3, time from OSA diagnosis, diagnostic criteria and severity of OSA, related medical history, comorbid conditions, concomitant medication and other sleep treatments.

4. Interventions: type of surgery, pharmacologic treatments, watchful waiting or no intervention.

5. Outcomes: instrument or scale for measurement, follow-up length.

6. Results: point estimates and measures of variability for continuous variables, frequency counts for dichotomous variables, number of patients.

\section{Risk of bias assessment}

At least two review authors will assess the risk of bias of each included study independently and in duplicate. RCTs will be assessed using the Cochrane Risk of Bias tool, which include seven domains: random sequence generation, allocation concealment, blinding of participants and personnel, blinding of outcome assessment, incomplete outcome data, selective reporting and other bias. ${ }^{28}$ Risk Of Bias In Non-randomised Studies-of Interventions will be used to assess the risk of bias of cohort studies for seven domains: confounding, selection of participants into the study, classification of interventions, deviations from intended interventions, missing data, measurement of outcomes and selection of the reported result. ${ }^{29}$ According to the guidance for these assessment tools, RCTs will be judged for having a low, high or unclear risk of bias; cohort studies will be judged for having a low, moderate, serious, critical risk of bias or 'no information'.

\section{Measures of treatment effect}

Where we identify a sufficient number of studies with homogeneous populations and characteristics, we will carry out meta-analyses of primary and secondary outcomes. For the analysis of dichotomous outcomes, we will use risk ratios with $95 \%$ CIs; for the analysis of continuous outcomes, we will use the mean difference or standardised mean difference and the corresponding 95\% CIs. 


\section{Missing data}

Where necessary, we will contact the authors of studies to obtain missing data/information. For missing SD, we will use the methods detailed in section 7.7.3 of the Cochrane Handbook to impute them. ${ }^{28}$ In the Discussion section of the review, the potential impact of any missing data will be discussed.

\section{Assessment of reporting biases}

Where there are more than 10 studies that can be combined into a meta-analysis, we will use a funnel plot to explore the possibility of small study effects, which may indicate publication bias.

\section{Data synthesis}

Analyses will be performed with the Review Manager (RevMan) V.5.3 software. Meta-analyses will be performed using a random-effects model. The heterogeneity among studies included in each meta-analysis will be assessed with the $Q$ test statistic and $\mathrm{I}^{2}$ statistics. Data from RCTs and those from cohort studies will not be combined in any meta-analyses.

\section{Subgroup analysis and investigation of heterogeneity}

We will attempt to perform the following subgroup analyses:

1. Study design (eg, RCTs vs cohort studies).

2. Nutritional status of the patients (eg, obese vs nonobese).

3. Methods used to diagnose OSAHS (eg, polysomnographically confirmed OSAHS vs diagnosed OSAHS based on subjective measurements only).

4. Surgical procedures (eg, temperature controlled radiofrequency tonsillectomy and adenoidectomy, and complete tonsillectomy and adenoidectomy).

5. Time point of outcome measurement (eg, long-term vs short-term).

6. Scales used to measure outcome.

7. Definition of outcomes.

8. Age groups.

\section{Sensitivity analyses}

If a sufficient number of studies are included in this review, we will perform sensitivity analyses to assess the consistency and robustness of our results. When sufficient data are available, we will perform sensitivity analyses by:

1. Including only studies with low risk of bias.

2. Excluding studies in which the majority of enrolled subjects were above 18 .

3. Repeating data syntheses using a fixed-effect model.

\section{Publication bias}

Possible publication bias will be investigated with a funnel plot if at least 10 studies are included in a meta-analysis.

\section{Summary of findings tables}

We will use the Grades of Recommendation, Assessment, Development and Evaluation approach ${ }^{30} 31$ to assess the certainty of the supporting evidence behind each main comparison. In this process, RCTs will start as high certainty evidence and observational studies as low certainty evidence. Thereafter, five factors (risk of bias, imprecision, inconsistency, indirectness and publication bias) may lead to rating down the certainty of evidence, and three factors (large effect, dose response and all plausible confounding would reduce a demonstrated effect) may lead to rating up.

\section{Patient and public involvement}

The present work is based on a review of relevant studies and does not include original patient data. Therefore, no patients or public were involved in this review protocol.

\section{ETHICS AND DISSEMINATION}

Ethical approval is not required. The results of this study will be disseminated via peer-reviewed publications and social networks.

\section{DISCUSSION}

Nowadays, OSAHS is a common problem among children. In 2002, the American Academy of Pediatrics identified growth failure as one of the serious complications of untreated OSAHS. ${ }^{32}$ Many studies have indicated that growth failure can be reversed after AT. ${ }^{25}$ However, a recent RCT with a 7-month follow-up showed that the changes in height and height $\mathrm{z}$ score, as well as height velocity measures were similar between the early AT group and watchful waiting control. ${ }^{22}$ Therefore, the present systematic review will analyse the association between AT children's height, weight and growth-related biomarkers. To our knowledge, there has been no systematic review regarding this clinical question since 2009.

In this review protocol, we have planned to perform subgroup analyses on different age groups, different nutritional status and different follow-up lengths. First of all, growth failure appears to be more prevalent among younger children with OSAHS. Vontetsianos and colleagues ${ }^{33}$ found that height was improved only in children under the age of 5 years, and the "catch-up growth' was not possible if disadvantageous factors were not removed early in life. Therefore, AT performed at an earlier age may lead to a higher degree of growth acceleration, and early diagnosis and timely referral may be helpful for reducing the occurrences of OSAHS-related growth failures. Second, the previous literature has shown an association between nutritional status and growth, which makes paediatric obesity another potential confounding factor. ${ }^{34}{ }^{35}$ In addition, differences in the findings of relevant studies could be explained by different follow-up lengths. ${ }^{24}$

By summarising the current knowledge in the effects of AT on growth of children with OSAHS, this review can provide a comprehensive summary of the current evidence for clinicians, and help them achieve evidencebased practice when treating children with OSAHS. 
Author affiliations

${ }^{1}$ Hubei-MOST KLOS and KLOBM, School and Hospital of Stomatology, Wuhan University, Wuhan, China

${ }^{2}$ Centre for Evidence-Based Stomatology, School \& Hospital of Stomatology, Wuhan University, Wuhan, China

${ }^{3}$ Cochrane Oral Health, Division of Dentistry, School of Medical Sciences, Faculty of Biology, Medicine and Health, The University of Manchester, Manchester Academic Health Science Centre, Manchester, UK

${ }^{4}$ Department of Otorhinolaryngology-Head and Neck Surgery, Wuhan University Zhongnan Hospital, Wuhan, China

${ }^{5}$ Department of Developmental and Behavioral Pediatrics, Pediatric Translational Medicine Institution, Shanghai Children's Medical Center, Shanghai Jiaotong University School of Medicine, MOE-Shanghai Key Laboratory of Children's Environmental Health, Shanghai, China

Contributors $\mathrm{FH}$ and $\mathrm{HH}$ conceived of this review. $\mathrm{FH}$ and $\mathrm{TZ}$ developed the search strategy and drafted the manuscript. TW, QS, XC, HW, FJ and HH revised the manuscript. $\mathrm{HH}$ is the guarantor. All authors participated in the determination of eligibility criteria, the risk of bias assessment strategy and data extraction methods. All authors read and approved the final manuscript.

Funding This work was supported by the National Key Clinical Specialties Construction Program of China (No [2013]544), China Postdoctoral Science Foundation (No 2018M630884) and the Fundamental Research Funds for the Central Universities (No 2042018kf0070, Wuhan University). The funding sources had no involvement in the study design, collection, analysis and interpretation of data, preparation of the manuscript or in the decision to publish.

Competing interests None declared.

Patient consent for publication Not required.

Provenance and peer review Not commissioned; externally peer reviewed.

Open access This is an open access article distributed in accordance with the Creative Commons Attribution Non Commercial (CC BY-NC 4.0) license, which permits others to distribute, remix, adapt, build upon this work non-commercially, and license their derivative works on different terms, provided the original work is properly cited, appropriate credit is given, any changes made indicated, and the use is non-commercial. See: http://creativecommons.org/licenses/by-nc/4.0/.

\section{REFERENCES}

1. Marcus CL, Brooks LJ, Draper KA, et al. Diagnosis and management of childhood obstructive sleep apnea syndrome. Pediatrics 2012;130:576-84.

2. Alexander NS, Schroeder JW. Pediatric obstructive sleep apnea syndrome. Pediatr Clin North Am 2013;60:827-40.

3. Kitamura T, Miyazaki S, Kadotani H, et al. Prevalence of obstructive sleep apnea syndrome in Japanese elementary school children aged 6-8 years. Sleep Breath 2014;18:359-66.

4. Andersen IG, Holm J-C, Homøe P. Obstructive sleep apnea in children and adolescents with and without obesity. Eur Arch Otorhinolaryngol 2019;276:871-8.

5. Bonuck K, Parikh S, Bassila M. Growth failure and sleep disordered breathing: a review of the literature. Int J Pediatr Otorhinolaryngol 2006;70:769-78.

6. Smith DF, Vikani AR, Benke JR, et al. Weight gain after adenotonsillectomy is more common in young children. Otolaryngol Head Neck Surg 2013;148:488-93.

7. Gottlieb DJ, Chase C, Vezina RM, et al. Sleep-Disordered breathing symptoms are associated with poorer cognitive function in 5-year-old children. J Pediatr 2004;145:458-64.

8. Marcus CL, Greene MG, Carroll JL. Blood pressure in children with obstructive sleep apnea. Am J Respir Crit Care Med 1998;157:1098-103.

9. Kwok KL, Ng DKK, Cheung YF. BP and arterial distensibility in children with primary snoring. Chest 2003;123:1561-6.

10. Macari; AT, Haddad RV. The case for environmental etiology of malocclusion in modern civilizations - Airway morphology and facial growth 2016;22:223-33.

11. Becking BE, Verweij JP, Kalf-Scholte SM, et al. Impact of adenotonsillectomy on the dentofacial development of obstructed children: a systematic review and meta-analysis. Eur J Orthod 2017;39:509-18.

12. Marcus CL, Carroll JL, Koerner CB, et al. Determinants of growth in children with the obstructive sleep apnea syndrome. J Pediatr 1994;125:556-62.

13. Stradling JR, Thomas G, Warley AR, et al. Effect of adenotonsillectomy on nocturnal hypoxaemia, sleep disturbance, and symptoms in snoring children. Lancet 1990;335:249-53.

14. Cielo CM, Gungor A. Treatment options for pediatric obstructive sleep apnea. Curr Probl Pediatr Adolesc Health Care 2016;46:27-33.

15. Pavoni C, Cretella Lombardo E, Lione R, et al. Orthopaedic treatment effects of functional therapy on the sagittal pharyngeal dimensions in subjects with sleep-disordered breathing and class II malocclusion. Acta Otorhinolaryngol Ital 2017;37:479-85.

16. Pavoni C, Cretella Lombardo E, Franchi L, et al. Treatment and posttreatment effects of functional therapy on the sagittal pharyngeal dimensions in class II subjects. Int J Pediatr Otorhinolaryngol 2017;101:47-50.

17. Jazi SMH, Barati B, Kheradmand A. Treatment of adenotonsillar hypertrophy: a prospective randomized trial comparing azithromycin vs. fluticasone. J Res Med Sci 2011;16:1590-7.

18. Bar A, Tarasiuk A, Segev Y, et al. The effect of adenotonsillectomy on serum insulin-like growth factor-I and growth in children with obstructive sleep apnea syndrome. J Pediatr 1999;135:76-80.

19. Wijayasingam G, Deutsch P, Jindal M. Day case adenotonsillectomy for paediatric obstructive sleep apnoea: a review of the evidence. Eur Arch Otorhinolaryngol 2018;275:2203-8.

20. Wiley Online Library. Our experience. Coblation" intracapsular tonsillectomy (tonsillotomy) in children: a prospective study of 100 consecutive cases. Clinical Otolaryngology 2015;39:301-7.

21. Mitchell RB. Adenotonsillectomy for obstructive sleep apnea in children: outcome evaluated by pre- and postoperative polysomnography. Laryngoscope 2007;117:1844-54.

22. Katz ES, Moore RH, Rosen CL, et al. Growth after adenotonsillectomy for obstructive sleep apnea: an RCT. Pediatrics 2014;134:282-9.

23. Williams EF, Woo P, Miller R, et al. The effects of adenotonsillectomy on growth in young children. Otolaryngol Head Neck Surg 1991;104:509-16.

24. Tahara S, Hara H, Yamashita H. Evaluation of body growth in prepubertal Japanese children with obstructive sleep apnea after adenotonsillectomy over a long postoperative period. Int J Pediatr Otorhinolaryngol 2015;79:1806-9.

25. Bonuck KA, Freeman K, Henderson J. Growth and growth biomarker changes after adenotonsillectomy: systematic review and metaanalysis. Arch Dis Child 2009;94:83-91.

26. Shamseer L, Moher D, Clarke M, et al. Preferred reporting items for systematic review and meta-analysis protocols (PRISMA-P) 2015: elaboration and explanation. BMJ 2015;349:g7647.

27. Reeves BC DJ, Higgins JPT, Wells GA. Chapter 13: Including non-randomized studies. In: Higgins JPT, Green S, eds. Cochrane Handbook for systematic reviews of interventions version 510 (updated March 2011): the Cochrane collaboration, 2011.

28. Higgins J, Green S. Cochrane Handbook for systematic reviews of interventions version 5.1.0. The Cochrane Collaboration, 2011.

29. Sterne JAC, Hernán MA, Reeves BC, et al. ROBINS-I: a tool for assessing risk of bias in non-randomised studies of interventions. BMJ 2016;355.

30. Guyatt GH, Oxman AD, Schünemann HJ, et al. Grade guidelines: a new series of articles in the Journal of clinical epidemiology. J Clin Epidemiol 2011;64:380-2.

31. Hultcrantz M, Rind D, Akl EA, et al. The grade Working group clarifies the construct of certainty of evidence. J Clin Epidemiol 2017;87:4-13.

32. Section on Pediatric Pulmonology, Subcommittee on Obstructive Sleep Apnea Syndrome. American Academy of Pediatrics. Clinical practice guideline: diagnosis and management of childhood obstructive sleep apnea syndrome. Pediatrics 2002;109:704-12.

33. Vontetsianos HS, Davris SE, Christopoulos GD, et al. Improved somatic growth following adenoidectomy and tonsillectomy in young children. Possible pathogenetic mechanisms. Hormones 2005;4:49-54.

34. Tauman R, Gozal D. Obesity and obstructive sleep apnea in children. Paediatr Respir Rev 2006;7:247-59.

35. Soultan Z, Wadowski S, Rao M, et al. Effect of treating obstructive sleep apnea by tonsillectomy and/or adenoidectomy on obesity in children. Arch Pediatr Adolesc Med 1999;153:33-7. 